\title{
Probabilistic Methods for Drug Dissolution. Part 2. Modelling a Soluble Binary Drug Delivery System Dissolving in vitro
}

\author{
Ana Barat \\ Heather J. Ruskin \\ Martin Crane \\ School of Computing, Dublin City University, Dublin 9, Ireland
}

\begin{abstract}
The objective of this work is to use Direct Monte Carlo techniques in simulating drug delivery from compacts of complex composition, taking into consideration the special features of the dissolution in vitro environment. The paper focuses on simulating a binary system, consisting of poorly-soluble drug, dispersed in a matrix of highly-soluble acid excipient. At dissolution, the acid excipient develops certain mechanisms, based on local $\mathrm{pH}$ modifications of the medium, which strongly influence drug release. Our model directly accounts for such effects as local interactions of the dissolving components, development of wall-roughness at the solid-liquid interface, moving concentration boundary layer and mass transport by advection. Results agree with experimental data and have demonstrated that when modelling dissolution in vitro, special attention must be paid to including the particular conditions of the dissolution environment.
\end{abstract}

KEYWORDS

Modelling; Drug delivery systems; Dissolution; Design and Experiment; Multicomponent soluble compacts; Monte Carlo; Cellular Automata

\section{Introduction}

In vitro dissolution testing is important in designing, developing and testing new formulations, $[23,6]$. In order to achieve the appropriate concentrations of the desired drug in vivo, (i.e. in the target organs and tissues), during the desired period of time, the dissolution profiles in vitro need to satisfy certain criteria, generally established by the pharmacopoeias $[24,6]$. Thus dissolution in vitro can be regarded as the first step toward modelling in vivo dissolution and absorption. The dissolution rate is measured, in practise, using one of a number of standard dissolution test methods outlined in international pharmacopoeias, such as the European Pharmacopoeia (Ph Eur) and United States Pharmacopoeia (USP). One commonly used dissolution test apparatus is the Paddle Dissolution Apparatus (see Figure 1), known as Apparatus 2 [1].

However, there is a number of difficulties related to in vitro dissolution testing. Very often, the relation between the formulation and process parameters of a pharmacological compact, and its required in vitro dissolution profile, is not entirely understood, due to the complexity of mass transport at dissolution. Complex mass transport often results in barely tractable effects, like interactions and synergies. For these reasons, experimentation associated with the field of drug design is very costly and time consuming. Thus, modelling the drug release can increase the performance in the design of new products, by on the one hand, making predictions and selecting the best candidate parameters to be experimentally tested, and, on the other hand, helping to develop scientific understanding of the phenomena involved in the dissolution process.

Many different modelling approaches to drug dissolution have been taken throughout the last decades, with mathematical modelling prevailing $[23,22,18,4]$. Other, less traditional alternative methods, like stochastic approaches, [2], direct Monte Carlo (MC) methods, [9, 8, 25, 13, 15, 14], 


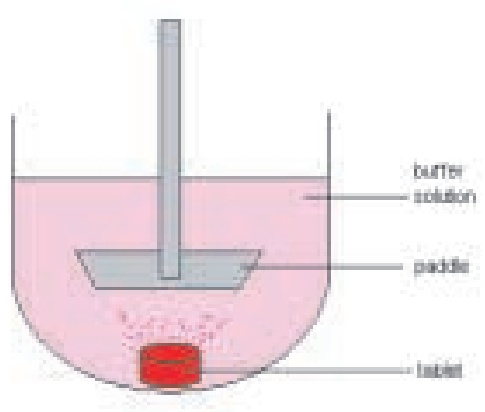

Figure 1: Schematic representation of the USP (United States Pharmacopoeia) Paddle Apparatus.

artificial neural networks (ANN) and genetic algorithms (GA) [24], have also recently been considered. These methods, used in parallel with the traditional ones, bring complementary advantages, such as the possibility of investigating the microscopic aspects of the problem in the case of MC methods, and optimisation schemes in the case of ANN and GA.

Previous research has indicated that direct MC could be a valuable tool in the field of modelling the dissolution of drug delivery systems characterised by complex internal structure, $[9,25,15]$.

Monte Carlo (MC) methods are used for solving various kinds of computational problems using random pseudo-random numbers. $\mathrm{MC}$ is extremely important in computational physics and related applied fields, because phenomena, which are difficult to quantify directly, can be treated as distributions of random numbers. Monte Carlo simulations have been considered for solving different problems associated with multiple-particle systems. Very often in these cases, Monte Carlo techniques have been applied in the framework of Cellular Automata, as shown in Part 1. Previous to being used to simulate systems such as those involving drug dissolution and delivery, the combination of direct Monte Carlo techniques and Cellular Automata have proved useful in the study to many other kinds of systems exhibiting complex behaviour [3, 16].

In this paper we explore the possibilities of MC modelling, for investigating the in vitro dissolution of a particular class of compacts, (used as model drug delivery systems in Healy and Corrigan [11]). The behaviour exhibited by these compacts in reactive media seems to be difficult to predict. Besides diffusion investigation, the model is designed to capture the particular features of the in vitro environment intrinsic to a dissolution paddle apparatus. The dissolution profiles generated by the Monte Carlo approach were found to be in accord with experimental observations on the dissolution of ibuprofen/acid excipient model drug delivery systems.

The following two sections are dedicated to the presentation of the problem and the solutions for solving different aspects of it, proposed in the past. The last section presents our MC model solution.

\section{Multicomponent soluble compacts and in vitro environ- ment}

\subsection{Binary compacts}

In the area of modelling dissolution of drug delivery systems, multicomponent soluble systems have not received enough attention, despite the fact that solid dosage forms invariably contain multiple soluble components [20]. Several theoretical approaches to describing binary systems are due to 
Ramtoola and Corrigan [20] and references therein. In the case of non-ionizable binary systems, where the components have different solubilities $\left(C_{s_{x}}\right.$ and $\left.C_{s_{y}}\right)$ and different diffusion coefficients $\left(D_{x}\right.$ and $\left.D_{y}\right)$, at the start of the diffusion process, the two components tend to dissolve at rates proportional to their diffusion coefficients. Later on, only one of the components will generally remain at the solid-liquid interface. The other component will have to dissolve through the porous system formed inside the less soluble component. Only at the critical mixture ratio, defined by:

$$
\frac{N_{x}}{N_{y}}=\frac{D_{x} C_{s x}}{D_{y} C_{s y}}
$$

will there be no porous layer formed at the surface and both components will dissolve at a rate equal to that of the pure component. In each case, $N_{x}$ and $N_{y}$ are the original amounts of components $x$ and $y$. In the general case, the dissolution rates of the components are calculated according to Fick's first law [5]. When a steady state is reached, the limiting dissolution rate of the component which remains at the surface is given by:

$$
G_{x}=\frac{D_{x}}{h} C_{s x}
$$

while the dissolution rate of the receding component $y$ is given by:

$$
G_{y}=\frac{D_{y} C_{s y}}{\left(h+\frac{\tau}{\varepsilon}\right)\left(s_{1}-s_{2}\right)}
$$

where $h$ is the thickness of the diffusion film, $\tau$ is the tortuosity, referring to the complexity of the system of channels which forms in $3 \mathrm{D}, \varepsilon$ is the porosity (often unknown though) and $\left(s_{1}-s_{2}\right.$ ) is the thickness of the porous layer formed at the solid-liquid interface. In the case where the components are ionizable the situation is more complex, [20]. In experiments on the dissolution of benzoic and salicylic acids in buffered media, the authors found that the dissolution rates, particularly for benzoic acid at intermediate weight fractions, were lower than theoretical rates and explained these in terms of surface $\mathrm{pH}$ effects.

Subsequently, Healy and Corrigan $[11,12]$ have conducted experimental and theoretical studies on the dissolution of soluble ibuprofen/acidic excipient compressed mixtures in reactive media, using model compacts. These were obtained by first grinding the two components in powders, mixing the products, and finally compressing them. On dissolution in reactive media, the compacts exhibit complex behaviour, in spite of their binary composition. The authors have presented a modified model for dissolution of binary systems of ibuprofen and various acid excipients. The predicted dissolution rates for the excipients tend to be higher than those obtained experimentally in the case of more soluble acids.

\subsection{Introduction to the in vitro dissolution medium}

\subsubsection{Dissolution apparatuses}

As mentioned before, one of the specific interests of this paper is to take into consideration the in vitro environment used for dissolution testing, because the settings of the dissolution apparatuses are known to affect the process of compact dissolution. In Healy and Corrigan, 1992 [11], the in vitro environment consisted of a water-jacketed flat-bottomed dissolution apparatus [10], and in Healy and Corrigan, 1996 [12], a more standard dissolution apparatus of USP type 2 has been used (see Figure 1).

A standard dissolution apparatus consists of a container, filled with the dissolution medium, with the compact situated at the bottom of the apparatus (see Figure 1). The dimensions of 
the apparatus are such that we can make the assumption that the solvent inside has sink or close-to-sink properties, (i.e. the system behaves as if the tablet dissolves in an infinite solvent). ${ }^{1}$

A paddle is used to stir the buffer solution at a constant rate. The stream created produces a velocity boundary layer around the compact. There are two mechanisms of mass transport in the system: diffusion and advection, ${ }^{2}$ the latter due to the component of the stream velocity oriented along the surface of the compact. This component is responsible for carrying away quantities of matter proportional to it's strength. One of the targets of the present work was to build into our Monte Carlo model both mechanisms of transport: both diffusion and advection.

\subsubsection{Velocity boundary layer and concentration boundary layer}

The hydrodynamic conditions of the in vitro environment are such that the velocity boundary layer at the top of a cylindrical compact is very difficult to describe. Crane et al. [7,6] investigated the velocity boundary layer, as well as the concentration boundary layer formed around the curved surface of a cylindrical compact in the USP apparatus, in order to better understand the concentration profiles which form at the solid-liquid interface on dissolution.

In this study, the component of the fluid flow, parallel to the curved surface of the cylinder, is examined. Far away from the compact, the fluid flows at its maximum speed: $\vec{U}_{0}$, but approaching the solid-liquid interface, the velocity decreases to zero at the surface of the solid, where the "nonslip" condition holds. Hence, the term of velocity boundary layer refers to the small region of space close to the solid immersed into the flowing liquid, where the flow velocity varies from zero to it's maximum $\vec{U}_{0}$ (see Figure 2, a).

As the solid dissolves, a concentration profile is established at the solid-liquid interface. Since the ratio of the diffusivity to the kinematic viscosity of the fluid (i.e. of the buffer solution), $\frac{D}{\nu}$, is very much less than unity, the diffusion boundary layer thickness $\delta_{c}$ is smaller than the velocity boundary layer thickness $\delta$, [7]. For the immediate solid-liquid interface, in contrast to the velocity profile, the concentration is at saturation, decreasing to zero with the distance from the surface (see Figure $2 \mathrm{~b}$ ). Thus, by the term concentration boundary layer, we mean the region of solution in the solid-liquid interface vicinity, where the concentration of solute in the solvent is greater than zero. The thicknesses of velocity/concentration boundary layers vary with the distance from the leading edge of the compact (Figure 2).

\subsubsection{Mathematics for boundary layers}

The velocity boundary layer thickness for a flat plate in an axial flow is given by the solution of Blasius equation [19, 21]:

$$
\delta=3.4 \sqrt{\frac{x \nu}{U_{0}}}
$$

where $x$ is the distance from the leading edge of the compact (Figure 2) and $\nu$ and $U_{0}$ are defined as previously.

Equation (5) gives the relation between $\delta_{c}$ and $\delta$ :

$$
\frac{\delta}{\delta_{c}}=S_{c}^{\frac{1}{3}}
$$

where $S_{c}$ is the Schmidt number, defined as:

\footnotetext{
${ }^{1}$ This means that far away from the dissolving compact, the concentration of solute in the solvent can be approximated by zero.

${ }^{2}$ The term "advection" refers to the transport of material from one region to another.
} 

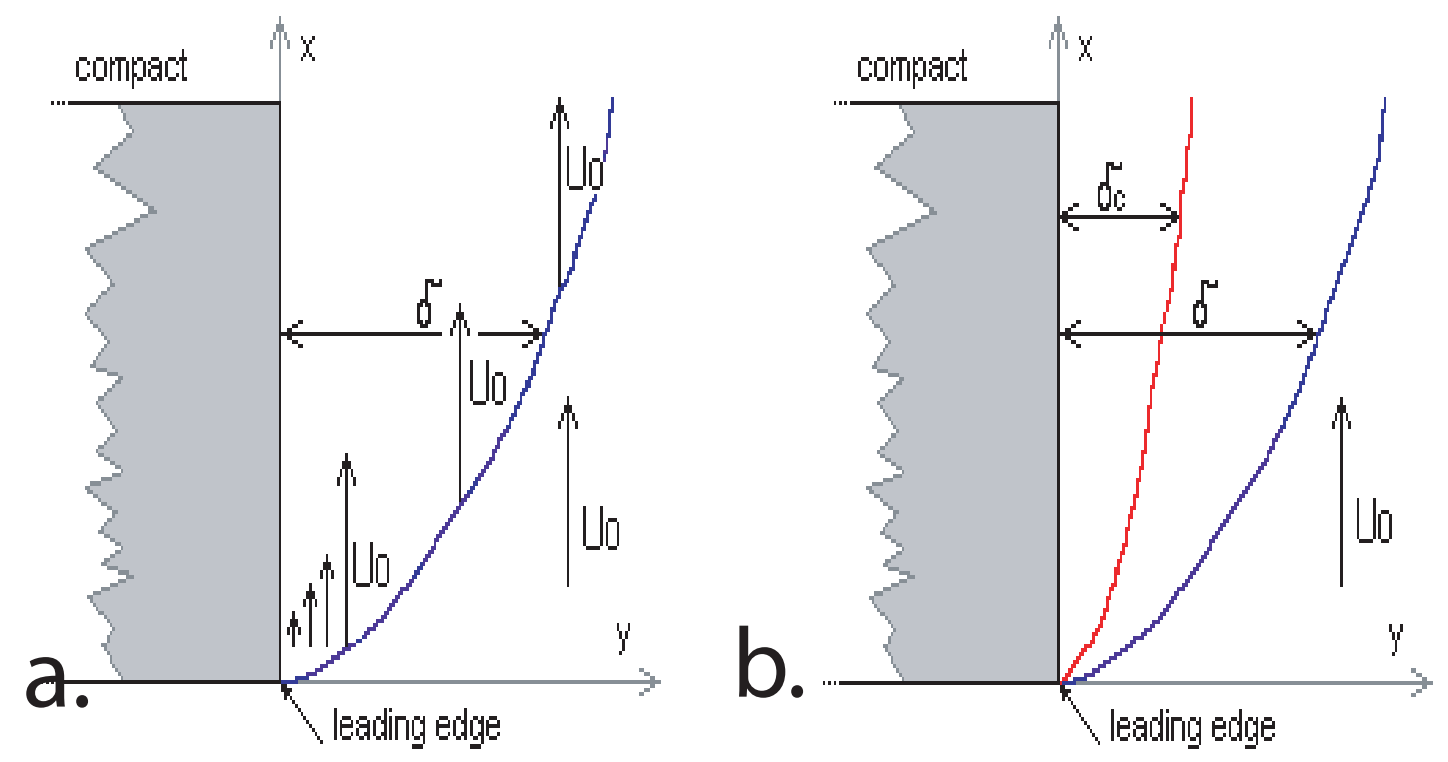

Figure 2: a. The velocity boundary layer of a non-dissolving solid immersed in a flowing liquid. $\delta$ is the thickness of the boundary layer, the blue curve indicates the points in the space where the velocity is at its maximum. b. Velocity and concentration boundary layers for a dissolving solid. $\delta_{c}$ is the thickness of the concentration boundary layer. The curve situated closer to the compact surface indicates the points in space where concentration of solute in solvent reaches low, close to zero, values.

$$
S_{c}=\frac{\nu}{D}
$$

Once the thickness of the concentration boundary layer has been computed, it is possible to compute the value $C^{*}$ of the maximum concentration at each point $(y, x)$, (Figure 2), within the boundary layer. One way to compute the concentration in the boundary layer is to use the Pohlhausen solution, which approximates the variation of concentration by either a polynomial, or, in the case of Crane et al. [6], by a sinusoid:

$$
\frac{C^{*}}{C_{\text {satur }}}=1-\sin \left(\frac{\pi y}{2 \delta_{c}}\right)
$$

Here, $C_{\text {satur }}$ is the saturation concentration of a given component, $C^{*}$ is the concentration at a given point $y$ within the concentration boundary layer and $\delta_{c}$ is the thickness of the boundary layer at a given height of the cylindrical compact.

From simplification considerations, the problem of in vitro radial dissolution was investigated in $[6,7]$ for simple layered binary systems (with 3 to 5 alternate layers, as in Figure 3). The investigation used numerical methods in combination with a Pohlhausen profile (see Equation (7)), to model the concentration boundary layer formed around the dissolving cylindrical device in the USP apparatus.

Numerical results, depicting the concentration profile within the boundary layer during a very short period of time, from Finite Element techniques, together with those from a semi-analytical Pohlhausen type approximation to the concentration boundary layer, have shown good agreement with experimental data. These studies have demonstrated that the conditions in vitro have an 


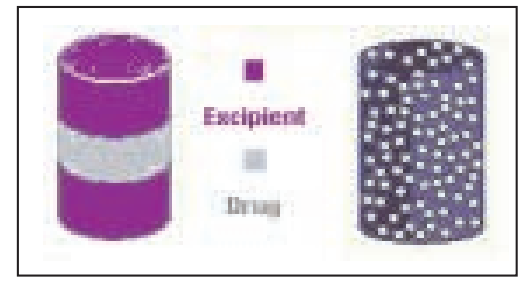

Figure 3: Layered compact versus matrix compact

a.

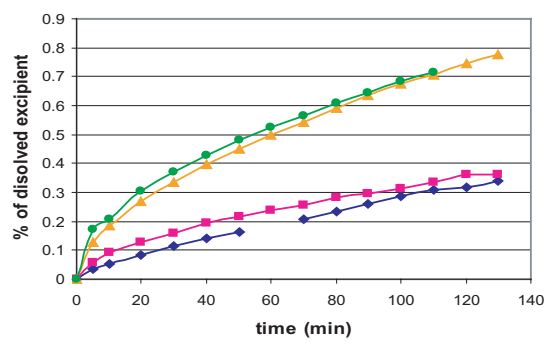

b.

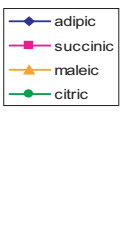

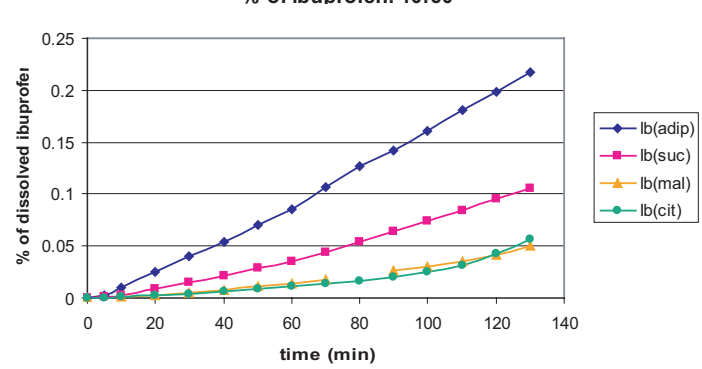

Figure 4: Experimental results

a. Dissolution profiles of the acid excipients from 40:60 ibuprofen/acid excipient compressed discs. b. Dissolution profiles of ibuprofen from 40:60 ibuprofen/acid excipient compressed discs. The vertical axis represents the mass fraction of released drug, i.e., the ibuprofen. Based on published data, [11].

important impact on the way in which a compact dissolves. However, the boundary layer for dissolving matrix compacts has not been considered yet. It seems that the matrix compacts exhibit physics which are less tractable by numerical methods.

\subsection{Experimental situation}

We use the data obtained by Healy and Corrigan [11] for the dissolution of ibuprofen and a wide range of acid excipients from mixed disks in reactive media, (phosphate buffer), as reference.

The compacts used in the experimentation were cylinders, obtained by compressing $250 \mathrm{mg}$ of powder, having a composition of drug and excipient on a weight-for-weight basis. Previous processing by grinding and sieving of the components permits control of particle size of both drug and excipient powders. The drug and the excipient have dramatically different solubilities and slightly different diffusivities, while the acidic excipient has an effect on the solubility of ibuprofen at dissolution. The compact dissolves according to the following mechanism: at the dissolution of the acid excipient, the $\mathrm{pH}$ of the buffer decreases and this has a suppressing effect over the solubility of the other component (the ibuprofen). The dissolution apparatus used was a waterjacketed flat-bottomed $450 \mathrm{ml}$ cylindrical glass vessel. $250 \mathrm{ml}$ medium was used. The medium was stirred with a 3-blade stirrer immersed to a depth of $2.5 \mathrm{~cm}[10]$.

As an example, Figure 4 represents the dissolution of different cylindrical binary matrix systems: all of them contain ibuprofen (Solubility $=6.3 \mathrm{mg} / \mathrm{ml},[10]$ ) and an acid excipient of much higher solubility (see Table 1). The left part of Figure 4, (a) represents the dissolution profiles for four different acid excipients. The right of Figure 4, (b) shows the dissolution profiles for the drug, which is ibuprofen in all cases. It can be observed from Figure 4 that the more soluble the excipient, the more it suppresses the solubility, and therefore the release of ibuprofen. 


\begin{tabular}{|c|c|}
\hline Type of acid excipient & Solubility, $\mathrm{mg} / \mathrm{ml}$ \\
\hline \hline Adipic & 44.4 \\
\hline Succinic & 120.4 \\
\hline Maleic & 694 \\
\hline Tartaric & 764.5 \\
\hline Citric & 883.6 \\
\hline
\end{tabular}

Table 1: Solubilities of the acid excipients, from [10].

\section{Modelling}

\subsection{Main characteristics of the model}

In this investigation, we simulated the dissolution of the binary soluble system presented in the previous sections, using a 2D lattice and a Monte Carlo algorithm, as described below. The model specifically takes into consideration both the diffusion and the advection. The algorithm is implemented in $\mathrm{C}++$ and the library OpenGL has been used for the visualisation.

We represent the two solid species by particles with different properties. These particles can be moved on the sites of the lattice according to rules. In this particular case, we make the assumption that one species dissolves independently and is referred to as the excipient in the following, while the dissolution of the other species strongly depends on that of the former. We refer to this last species as the drug, to match the terminology of the experimental system we consider. The solvent is represented by empty sites. The state of a site $(i, j), \psi_{(i, j)}$ is defined by the quantity and type of the particles with which it is filled, i.e. the concentrations $C_{D}(i, j)$ and $C_{E}(i, j)$ of drug and excipient particles, respectively. The state of any site can evolve in time according to a function, which depends on the concentrations at the site itself and those in the local neighbourhood (Figure 12). The process of diffusion is simply simulated by the tendency of particles to move to adjacent sites according to specified rules. Figure 5 illustrates the possible states in the model and their schematic behaviour.

Our main target is to be able to predict the dissolved quantities of either of the two species, $M_{D}(t)$ and $M_{E}(t)$, at each time step.

\subsection{The initialisation}

The spatial configuration of the DDS is generated as follows:

- For each site of the lattice, which has to be occupied by "matter" in solid state, a random number uniformly distributed between 0 and 1 is generated.

- If this number is smaller than or equal to the proportion of drug, this site is considered as drug. It's state is assigned $\psi_{(i, j)}(t)=\mid D$, and the site is filled with a number $n_{D}$ of drug particles.

- Otherwise, the site is filled with $n_{E}$ excipient particles and $\psi_{(i, j)}(t)=\mid E$.

- In order to simulate the phenomena at the solid-liquid interface, the sites around the solid are assigned the liquid state: solution. We distinguish two types of solution states: (i) sites which are situated in the immediate proximity to the solid-liquid interface and which form the boundary layer with thickness $\delta_{c}$, (see Section 2.2): $\psi_{(i, j)}(t)=1-$, and (ii) those far enough from the dissolving compact and which behave as a $\operatorname{sink}^{3}: \psi_{(i, j)}(t)=\mid s$ (see Figure 5)

\footnotetext{
${ }^{3}$ The main difference between the boundary layer solution sites and the sink solution sites is that the first can receive and accommodate diffusing drug and excipient particles, whereas the second have the concentration of the diffusing species set to zero. This artifact comes from the assumption that the concentrations in the stirred liquid are so much smaller then the concentrations in the boundary layer, that these are approximated by zero: $C_{(i, j)}=0$
} 


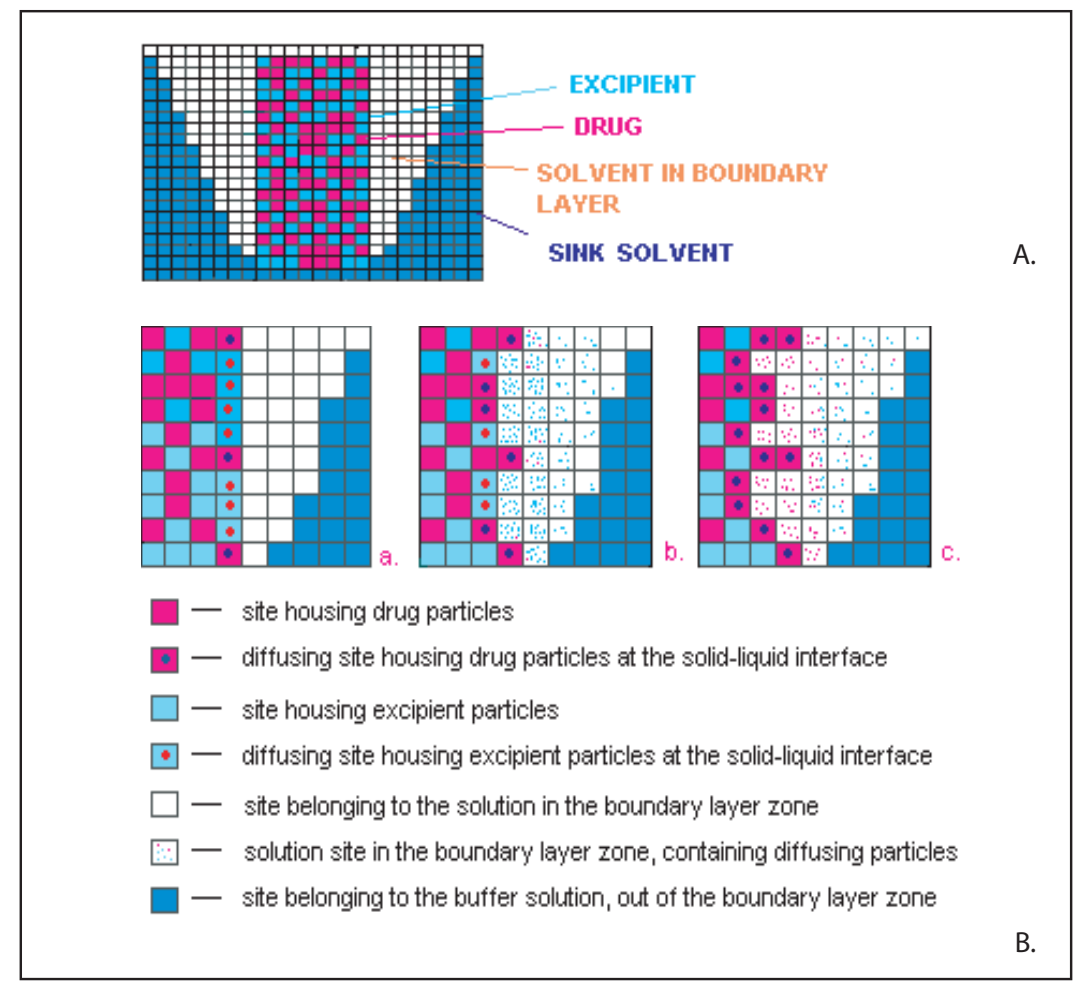

Figure 5: 2D model for a dissolving binary system.

a. Schematic representation of 2D model. The real dimensions are not respected in the picture. b. Simplified model of dissolution of a drug-excipient system. The grid represents a cylindrical tablet in longitudinal cut. The shots show the benefits of this kind of model for simulating cases where pores are formed inside a compact by the quick dissolution of one of the components. The second component has to dissolve through these pores and this process is different from dissolution at the surface.

- The sites in the solid which are exposed, through nearest neighbour connectivity, to solution sites, are attributed the following states: leaking drug and leaking excipient $\left(\psi_{(i, j)}(t)=1+\right.$ and $\left.\psi_{(i, j)}(t)=\mid x\right)$. From this instant, the particles from these sites will be allowed to move. The content in particles of leaking sites can only decrease.

\subsection{Modelling the diffusion}

Diffusion refers to the process by which molecules of different species intermingle as a result of their kinetic energy. In the model we represent the two solid species by particles. Since the components in the compact have highly different solubilities, they are expected to dissolve at different rates. For this reason, we allow for more then one particle to move to an adjacent site. The solubility of a given species is modelled by allowing not more than a fixed number of particles, $S=C_{M A X}$, on the sites considered as filled with solvent. The maximum allowed concentrations in the solvent, $C_{M A X_{\text {Drug }}}$ and $C_{M A X_{\text {Excipient }}}$, are proportional to the real solubilities. This means that the ratio $\frac{C_{M A X_{\text {Drug }}}}{C_{M A X_{\text {Excipient }}}}$ equals the solubility ratio $\frac{S_{D}}{S_{E}}$ between the drug and the excipient. In addition, the local solubility of a component depends on the local concentrations [11].

when $j=\delta_{c}$. When, in later stages, particles reach a sink solution site, they will be considered completely dissolved, thus they will be discarded from the system and their quantities - added to $M_{D}(t)$ and $M_{E}(t)$. 
Only the sites in the states $\psi_{(i, j)}(t)=\left|-, \psi_{(i, j)}(t)=\right|+$ and $\psi_{(i, j)}(t)=\mid x$ can diffuse. If the site $(i, j)$ is in one of the above states, the possibility of diffusion is considered by first looking at the kind of particles about to diffuse: dependent (drug) or independent (excipient) particles.

The decision to permit a batch of particles to diffuse is accepted or rejected by:

- Consulting a gradient-dependent probability, $p_{E}$ in the case of the excipient (Equation 8). In the following, $(i, j)$ symbolises the current site and $(i *, j *)$ - the adjacent site to which the possibility of diffusion is considered.

$$
p_{E}=\frac{C_{E(i, j)}-C_{E(i *, j *)}}{C_{E(i, j)}}
$$

- Consulting a probability, dependent on the excipient concentration in the neighbourhood, $p_{D_{0}}\left(p_{D_{0}}=f\left(\Sigma C_{E(i *, j *)}\right)\right)$ and a further, gradient-dependent probability, $p_{D_{1}}$ (Equation 9$)$ in the case of the drug particles about to diffuse.

$$
p_{D_{1}}=\frac{C_{D(i, j)}-C_{D(i *, j *)}}{C_{D(i, j)}}
$$

- The diffusion operation itself is performed by calculating the number of particles of excipient, $f_{E}$, and those of drug, $f_{D}$, which will move to each of the adjacent sites. The number of excipient particles which hop from the site $(i, j)$ to the site $(i *, j *)$ are given by:

$$
f_{E}=p_{E} * X_{E}
$$

where $X_{E}$ is a variable uniformly distributed between one particle and the maximum number of particles allowed on the site $(i *, j *)$. The quantity of the particles on a site is limited by the species solubility $C_{M A X}$, therefore

$$
\left.X_{E}=\mathcal{U}\left(1, C_{M A X_{E}}-C_{(i *, j *)_{E}}\right)\right)
$$

- A normal distribution can be used as well to calculate $X_{E}$.

In order to perform a synchronous updating of the diffusion operation, we compute for all the cells on the lattice the incoming and outgoing quantities, and only then update the actual concentrations and increment the Monte-Carlo time step. After the update, all the particles which diffuse to sites in a state of sink solution $\psi_{(i, j)}(t)=\mid s$, are considered to be completely dissolved and are discarded from the system. The values of $M_{D}(t)$ and $M_{E}(t)$ are incremented respectively by the number of either kind of particles discarded from the system.

\subsection{Modelling the advection}

As mentioned, advection plays an important role in mass transport in the in vitro environment of the apparatuses used for dissolution testing. The flow created in these is very complex $[7,6,17]$. In this model we make the assumption that there is always a stream component which is oriented parallel to the surface of the compact, exposed to the dissolution medium.

If we do simulations in which the size of the site is much smaller then the thickness of the diffusion boundary layer, the advection operation can be performed using the Pohlhausen concentration profile (see Section 2.2) in a discretized form, adapted for our lattice model: $\left(C_{E(i, j)}^{*}\right.$, $\left.\psi_{(i, j)}(t)=\mid-\right)$. We make the assumption that a velocity/concentration boundary layer is established at the liquid/solid interface. At the surface the velocity is zero and thus the concentrations of all the diffusing species are at saturation. Therefore, in the immediate proximity to the solid surface, there are only diffusion-based phenomena. As we move away from the surface, the velocity increases from zero to it's maximum $\vec{U}_{0}$. At the same time, the concentration decreases, because 
the flow is responsible for carrying away quantities of matter proportional to the velocity of the stream.

In order to simulate the advection process, we consider every solution site within the boundary layer and the number of particles of drug and excipient $a_{D(i, j)}$ and $a_{E(i, j)}$ which will be carried away from it by the stream, to symbolise the mass transport property. The quantities $a_{D(i, j)}$ and $a_{E(i, j)}$ are computed so that, after they will have been discarded from the system, a concentration Pohlhausen profile (Equation 7) in a discretized form appears in the boundary layer region.

After an advection step is performed, there are cells from the boundary layer, in solution state, which have the outgoing quantities of particles $a_{D(i, j)}$ and $a_{E(i, j)}$, different from zero. The corresponding update operation consists of a sweep of the lattice which finds and discards these particles from the system, symbolising the mass transport due to advection. These values are added respectively to $M_{D}(t)$ and $M_{E}(t)$, while $a_{D(i, j)}$ and $a_{E(i, j)}$ are re-set to zero.

As the tablet dissolves, the boundary layer recedes in the space.

In the case in which the size of a site is in the same order of magnitude as the thickness of the diffusion boundary layer, the advection mass transfer can be performed by inverse Monte Carlo simulations. We consider that diffusion in the pores and diffusion at the surface of the solid does not happen in the same way. At the surface, larger $X_{E}$ than in Equation 11 (see previous section) are allowed to diffuse to adjacent sites. A normal distribution can be used in this case: $X_{E}=N(\mu, \sigma)$, where $\mu$ is the mean and $\sigma$ - the standard deviation of the distribution. In order to find out $\mu$ and $\sigma$, it is possible to:

- Sample values for $\mu$ and $\sigma: \mu_{i}$ and $\sigma_{i}$ - from, for example, uniform distributions.

- Use these values to perform a simulation and generate a dissolution profile.

- Compare the dissolution profile obtained to the real data.

- Accept $\mu_{i}$ and $\sigma_{i}$ if the results of the simulations are satisfactory.

- Reject $\mu_{i}$ and $\sigma_{i}$ them in case of unsatisfactory results, re-sample other values, $\mu_{i+1}$ and $\sigma_{i+1}$, and repeat the mentioned steps.

\subsection{Updating}

Before beginning any mass transport operation, the following update is performed:

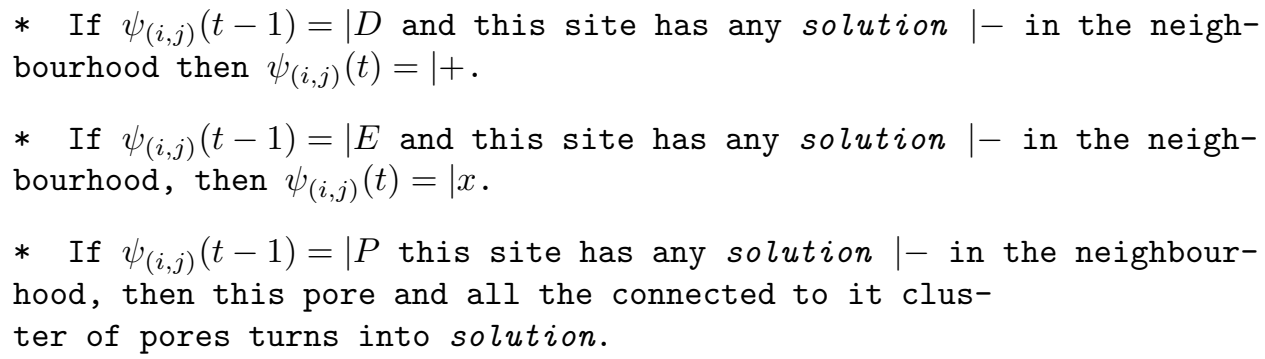

If the concentration of a leaking site decreases under the solubility $\left(C_{M A X_{D}}\right.$ or $\left.C_{M A X_{E}}\right)$, its state is updated to the solution state. This implies that the particles at the site are completely dissolved and that the site can begin to receive new particles:

$$
\begin{aligned}
& * \quad \text { If }\left\{\begin{array}{c}
\psi_{(i, j)}(t-1)=\mid+ \\
C_{D(i, j)} \leqslant C_{M A X_{D}}
\end{array} \text { then } \psi_{(i, j)}(t)=\mid-.\right. \\
& * \quad \text { If }\left\{\begin{array}{l}
\psi_{(i, j)}(t-1)=\mid x \\
C_{E(i, j)} \leqslant C_{M A X_{E}}
\end{array} \text { then } \psi_{(i, j)}(t)=\mid-.\right.
\end{aligned}
$$

After the updating phase, the diffusion can begin. 


\section{Results and discussion}

A MC time step, corresponding to time $t$, is a sequence of applying the operations of diffusion, advection and updating to all the sites from the lattice. The system is left to evolve during many $\mathrm{MC}$ iterations, incrementing the time value after each iteration. Samples of $M_{D}(t)$ and $M_{E}(t)$ are taken periodically, in order to plot their profiles at the end of the simulation.

We average simulation results on a certain number (25 in the following) of initial configurations of the device, because the spatial packing of the two components in the compact has some slight effect on the dissolution results.

In this section we analyse the capacity of the model described above to predict correct effects of the design parameters, like initial drug loading, initial porosity, form of the compact etc., on the dissolution rates. Besides its purpose of conserving the behaviour encountered in the real problem (by the microscopic content of the rules specified), a computer solution is prone to develop and exhibit its own special effects. These may interfere with the relevant trends. We desire to investigate the extent to which the purely numerical phenomena may affect the results of the simulations.

\subsection{The effect of the of the acid excipient solubility}

The suppression effect of the acid excipient is indirectly modelled by defining rules for dramatically decreasing the diffusing probabilities of the drug in the presence of local high concentrations of excipient. We have carried out simulations with binary systems where the properties of the dependent species (drug) have been kept the same for all the experiments with different solubility of the independent and more soluble species (excipient). The excipient received a wide range of solubilities noted $S_{E}$.

Figure 6 compares the behaviour of the system for two different excipients $E_{1}$ and $E_{2}$, one with a high solubility and the other with lower solubility $S_{E 1}>S_{E 2}$. The properties of the dependent component, the drug, are kept constant during the two simulations and $S_{E 1}>S_{D}, S_{E 2}>S_{D}$. Figure 6, (a) and (c) shows the concentration profiles for, respectively, $E_{1}$ and $E_{2}$. In both cases irregular dissolution fronts are developed, but the extent of their roughness is obviously a function of the solubility of the excipient. The excipient influences the dissolution of the dependent species by filling the free space with its particles and consequently decreasing the local solubility of the drug. The higher the solubility of the excipient, the higher the capacity of a solution site to accommodate excipient particles.

For the case of $E_{1}$, it can be noticed that the excipient has strongly receded from the surface (Figure 6, (a)), and has created a porous layer, through which the poorly-soluble drug dissolves only in the region where the excipient concentrations are very low (Figure 6, (b)). The drug dissolves through the pores created by the dissolution of $E_{1}$. For $E_{2}$ very different behaviour is exhibited. The porous layer is much thinner than in the previous case and the drug boundary has receded inward to a greater extent. This type of behaviour of binary drug delivery systems has been observed and described by [20].

We have plotted the fraction of the dissolved of drug and excipient against the time, comparing the dissolution profiles of drug and excipient with those obtained in the experiments. The results reproduce very well the trends and effects occurring at the dissolution of real binary compacts, containing ibuprofen and acid excipient [11].

Figure 7 shows the drug and the excipient profiles for different solubilities and for two different drug loadings. The porosity of the matrix is kept constant. Every dissolution curve is obtained by averaging the results of a particular number of simulations (20) characterised by the same initial parameters but with different initial configurations of the compact, (the 2 species are randomly distributed in the compact for each simulation). The plots show that as the solubility of the excipient increases, the dissolution of the drug is more and more suppressed. As in experiment, a positive curvature for the drug and a negative curvature for the excipient are obtained.

With highly-soluble excipients, the dissolution of the drug is very slow in the beginning, and when almost all the excipient is dissolved, the drug changes its dissolution rate, as can be seen 


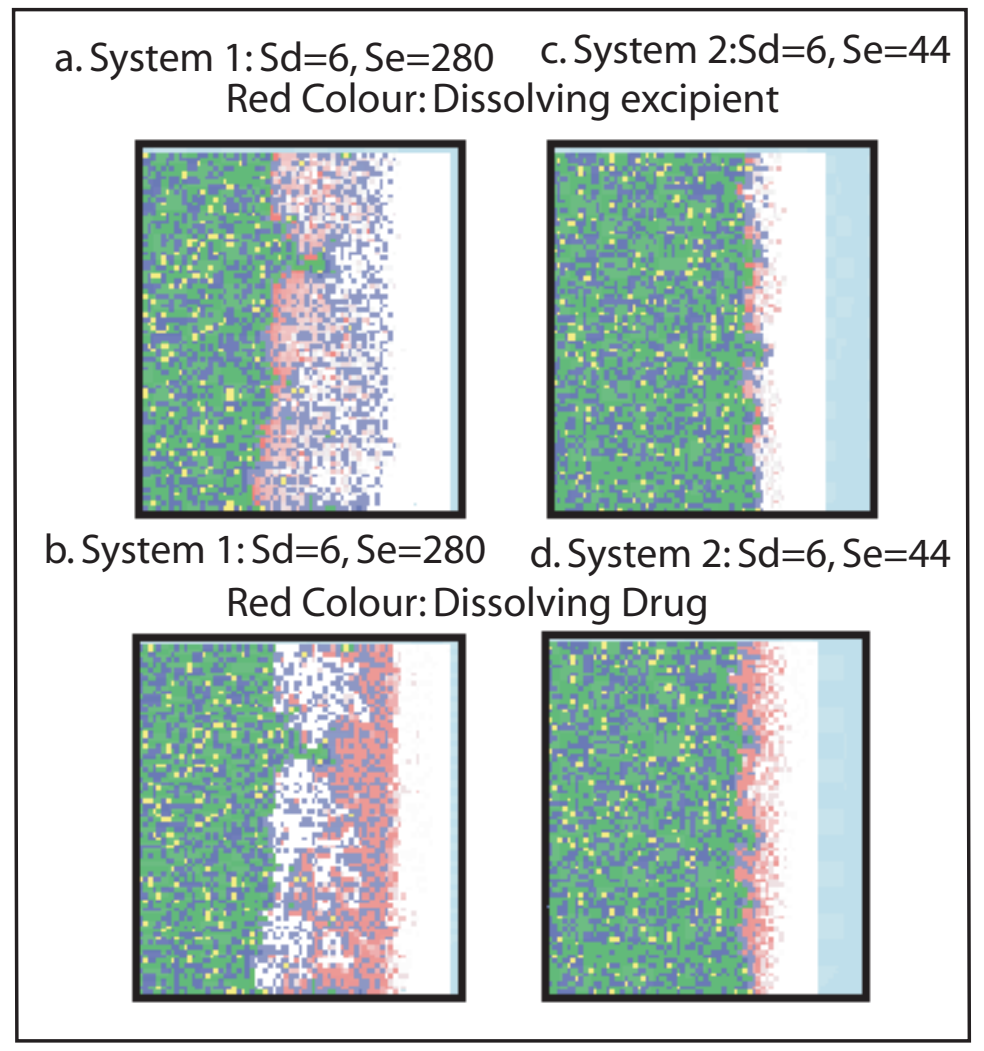

Figure 6:

Colours code: green - solid excipient, blue - solid drug, yellow - pores, white - solution in the boundary layer, clear blue - sink solution, shade of red in (a) and (c) - the concentration of excipient in the boundary layer solution, shade of red in (b) and (d) - the concentration of drug in the boundary layer solution. All the figures show the state of a 130/80 sites compact after 4000 generations. a. and b. System where the excipient has a relatively high solubility: $S_{E}=280$. c. and d. System where the excipient is less soluble: $S_{E}=44$. 


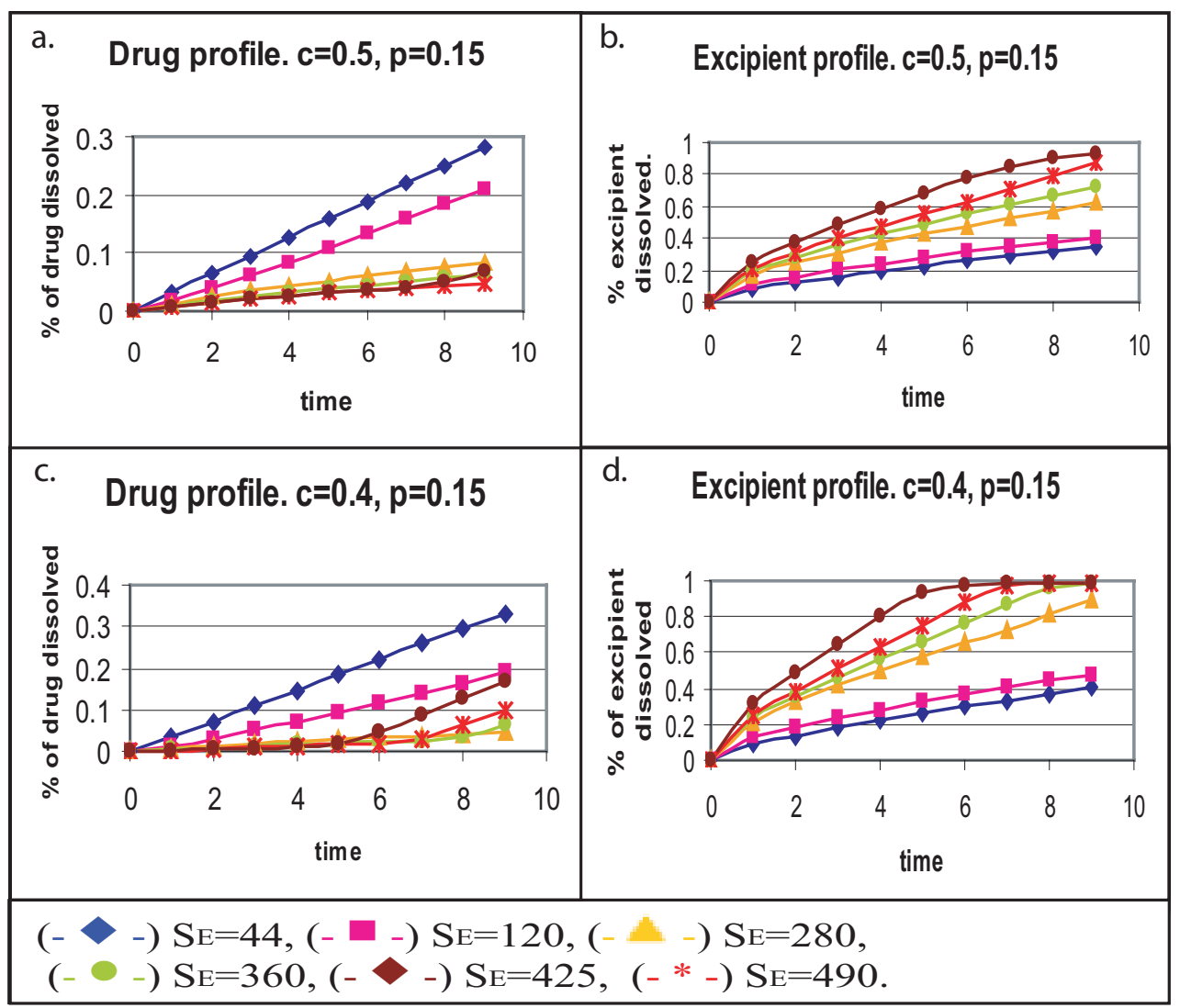

Figure 7: The effect of the solubility of the independent species on the dissolution of the dependent species

for some of the profiles in Figure 7, (c). In Figure 7 (c), the orange ibuprofen profile corresponds to the micro-environment represented on Figure 6 (c) and the blue profile shows the case of $E_{2}$, (Figure $6(\mathrm{~d})$ ).

We note that suppression of the advection step from the model leads to simulation results for the drug profiles which do not accord with experiment, hence environment-related features are important.

\subsection{Loading effect}

If we think of what effect the drug loading would produce in the beginning stages of the simulation, it is evident that the more drug the matrix contains, the less excipient is present, hence, the higher the dissolution rates for the drug. Figure 8 confirms this idea and shows more explicitly the effect of the initial loading of drug on the dissolution profile of the components. The same trend is observed in the experimental data: the greater the drug loading, the greater the fraction of the total drug mass released.

However if we produce simulations with an "excipient" of a lower solubility, like $S_{E}=C_{M A X_{E}}=$ 44, the opposite effect of the loading is observed (Figure 9). For $50 \%$ of drug loading, smaller fraction of total drug mass is released than in the case of $40 \%$ of drug.

This happens because the concentrations of excipient are not high enough to influence the drug dissolution profile. A matrix with lower initial drug loading contains a higher excipient loading. At its dissolution, the excipient creates a certain level of wall roughness, increasing the dissolution area for the drug and thus enhancing the drug dissolution, which is not suppressed by the relatively 


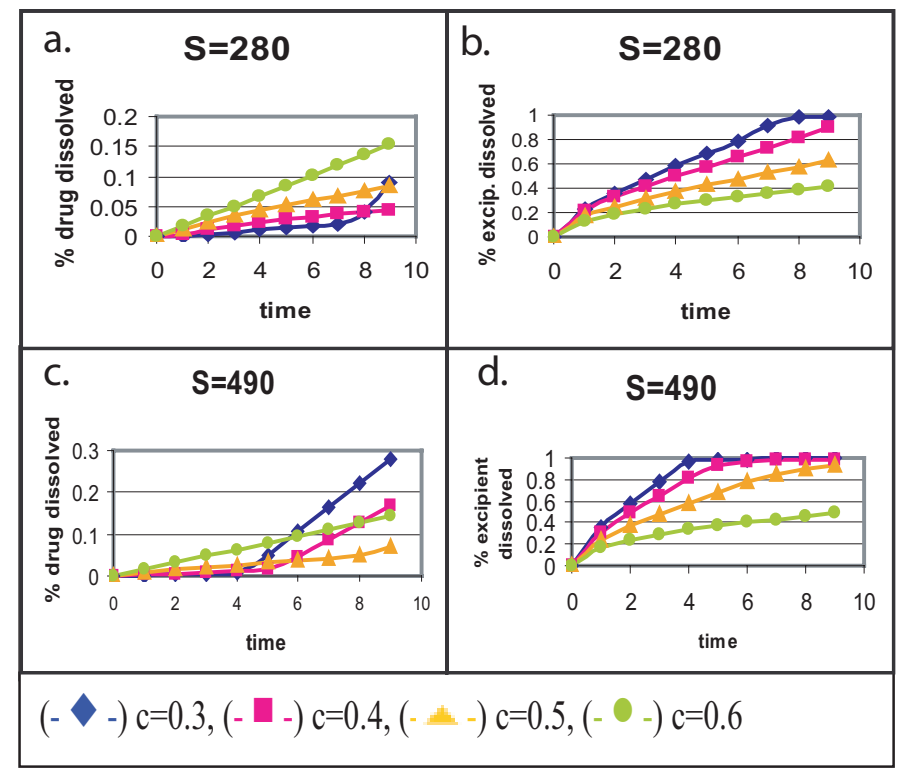

Figure 8: Loading effect for excipients with high solubility

low excipient concentrations.

When we have performed quantitative simulations (see Subsection 4.5), directly comparable to the experimental profiles, this inversion-effect appeared in the cases when running the model for mixtures of ibuprofen with adipic acid and, respectively, succinic acid (see Table 1), while the inversion does not appear in the experimental results. Why? Because effectively, the model generates too low excipient concentrations for the case of $50 \%$ of drug loading. The cause of this may be the modelling a 3D problem in 2D, as discussed in subsection 4.5). We have also found, when calibrating quantitative models with experimental data from dissolution of ibuprofen-only compacts, that the operation simulating advection, as set for generating the results from Figure 9 , is not enough "strong" to mimic the advection from the experimental part. After introducing a correction for the advection, the inversion effect has disappeared and agreement with the experimental data has been obtained.

Adipic acid is the acid with the lowest solubility (we can compare solubilities because the pKa values of all acids is very close), for which experimental data are available. The quantitative advection-corrected model suggests that the inversion effect reappears and increases for lower values of the excipient's solubility. Would this be confirmed in the reality if the corresponding components were available for experimentations?

\subsection{The effect of the initial porosity}

Figure 10 presents the simulation results for different porosities for dissolution of compacts containing very soluble excipients. The porosity value represents the fraction of sites belonging to the compact, which are free of particles. For the excipient, a higher porosity value enhances its dissolution rate. This effect grows stronger with increase in the excipient solubility, (Figure 10, (b) and (d)). In Figure 10, (d) the porosity factor disperses the dissolution profiles much more than in Figure 10, (b). On the other hand, the drug dissolution is suppressed more strongly at a higher porosity at the start of the dissolution phase. This is due to the suppression effect of the excipient, which dissolves well at high porosities.

Figure 11 shows the effects of the porosity on a system where the excipient is less soluble than in the previous case. The trends for the dissolution profiles for the excipient are the same, but less evident, as the profiles are not very dispersed. However, the effects of the factor porosity for the 


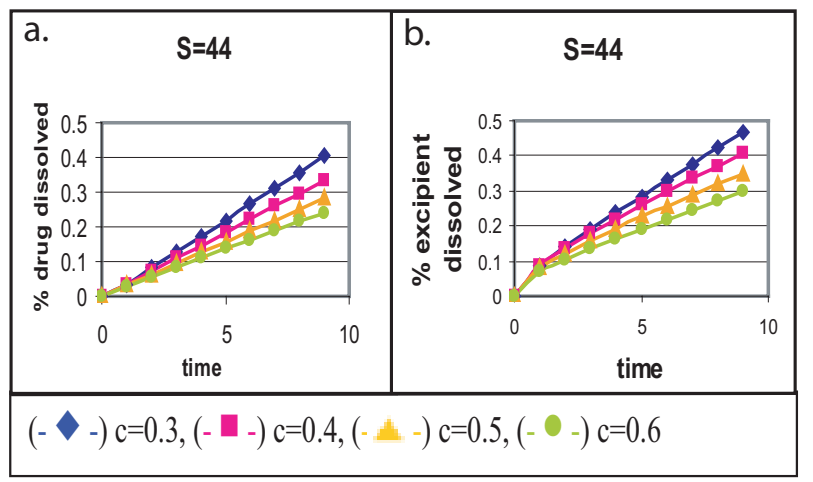

Figure 9: Loading effect for excipients with relatively low solubility a. Dissolution profiles for the drug. b. Dissolution profiles for the excipient.

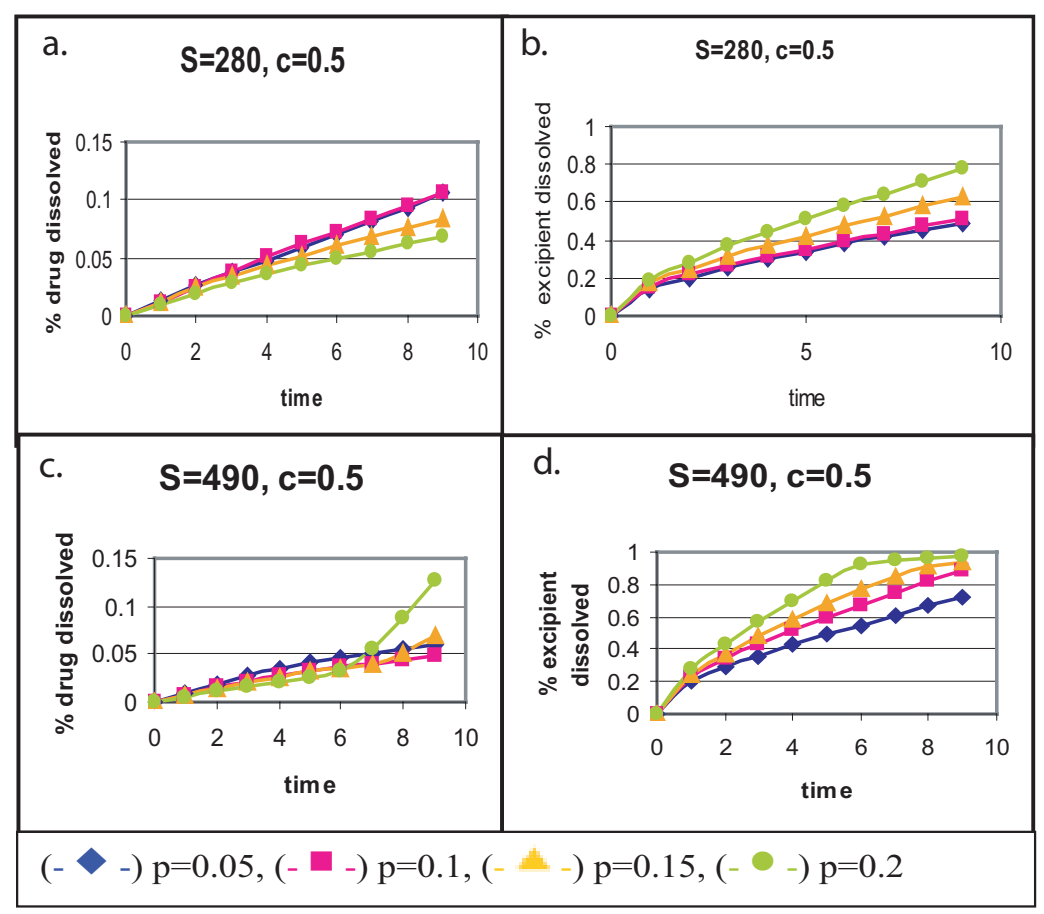

Figure 10: Porosity effect for excipients with high solubility 


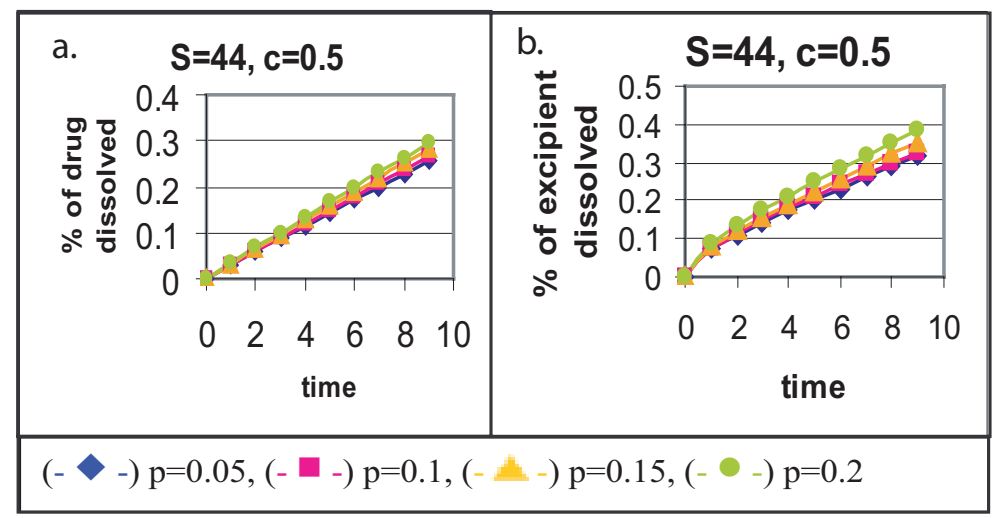

Figure 11: Porosity effect for excipients with relatively low solubility. a. Dissolution profiles for the drug from 50:50 drug/excipient matrices. b. Dissolution profiles for the excipient from 50:50 drug/excipient matrices.

drug differ from the previous case. Here, the higher the porosity, the higher the dissolution rate of the drug. This happens because, in this case, the drug exhibits a more independent behaviour. This means that, for this system, there is a solubility level of the excipient, for which its influence on the drug's dissolution is completely canceled or inverted. As explained for the loading effect, more experiments are necessary to completely verify whether the effects mentioned are totally model related, or not.

\subsection{Neighbourhood effect}

We find that different versions of the model are more or less adapted to simulating one or another case of the dissolution problem. Two different types of neighbourhoods were considered, (Figure 12, a), and we have found the Moore neighbourhood to be the most appropriate for our problem, because it permits for additional directions of particle movement in the $2 \mathrm{D}$ space, and this is evidently closer to reality. Von Neumann's neighbourhood provides good results for compacts containing more than $50 \%$ of excipient. When the quantity of the excipient in the tablet decreases, however, models using the Von Neumann neighbourhood, although showing quite good trends for the drug dissolution, strongly underestimate the quantities of dissolved excipient in the buffer solution, see Figure 12, (b). This is a model-related effect, and the stated under-estimations are due to the fact that sometimes the slowly dissolving drug sites completely block the excipient.

Models based on the Moore neighbourhood, with its eight nearest neighbours, provide simulations with less "trapped excipient" effects. The results presented in all the previous sections are obtained with the Moore Neighbourhood.

\subsection{Quantitative simulations}

The objective of the last part of the modelling was to pass from qualitative to quantitative simulations. As this paper is dedicated to the presentation of the problem and to the structure of the model, the work involved in the quantitative simulations is presented here just briefly. Mainly, quantifying the models consisted in:

- mapping the experimental binary system into 2D binary lattices, according to the mean size of the powder particles of drug and excipient.

- computing of the input parameters, such as number of particles per site, solubilities, the distribution of the number of excipient particles allowed to transfer to adjacent places, time of 

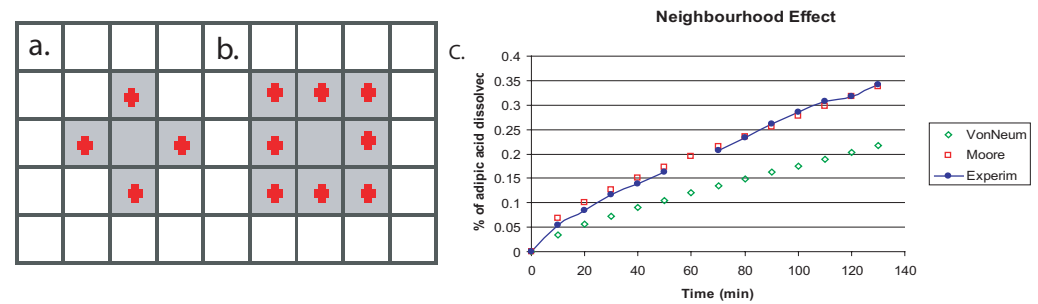

Figure 12: a. Von Neumann neighbourhood. b. Moore neighbourhood. c. Dissolution profiles of adipic acid from 40:60 mixed matrices. Continuous curve: experimental. Dots: Simulations. Here the quantitative model described in the next section has been used.

dissolution, time interval of sampling the quantities of interest, etc according to the physical properties of the 2 components, i.e. diffusion coefficients, densities ratios.

- calibrating the parameters, related to the advection process by inverse evaluation, (sampling, comparing to the experimental data, re-sampling).

- establishing the way different acid excipients suppress the dissolution of ibuprofen.

Figure 13 shows that the level of agreement achieved is quite good. The practical interest of a quantitative model is obvious. It made possible evaluating quantitatively parameters related to the porosity of the matrices. Part 1 presents more quantitative results.

Figure 14 shows a typical formulation for which the model is able to predict only the general trend and not so close values, like the ones in Figure 13. This happens for the cases where the drug loading is rather high. Two dimensions are not enough to model such configurations, because in the cases of $50 \%$ or more than $50 \%$ drug in $2 \mathrm{D}$, only few channels form and they are probably not enough for the excipient to evacuate. Presently, we work on 3D models which we hope to solve this kind of problem. Developing 3D models is interesting also because they will be able to provide values for the tortuosity. Expression 3 can then be used to calculate the dissolution rates. These dissolution rates can then be compared to the dissolution rates provided directly by the models and the experimental dissolution rates.

\section{Conclusions}

With our simple Monte Carlo model, direct consideration is taken of aspects such as timedependent porosity and thickness of porous layer, dissolution through pores, effects of particle size and distribution, concentration dependent solubilities, receding solid-liquid interface, dissolution over long periods of time and advection due to the in vitro environment. Thus, essential features, necessary to reproduce the complexity observed in the real world problem of in vitro drug dissolution, are captured. We note that suppression of the advection step from the model leads to simulation results for the drug profiles which do not accord with experiment, hence environmentrelated features are important.

The model can also easily be modified to simulate multicomponent compacts; for example, it can be used to see what happens if a mixture of ibuprofen/acid excipient is compressed in an inert non-soluble matrix of ethylcellulose, (a material used in the present day in the manufacturing of controlled release systems). In this case, it is important to examine the percolation phenomena and the MC model has the advantage of permitting such type of investigations. 
a. Ibuprofen/Succinic acid. 40:60

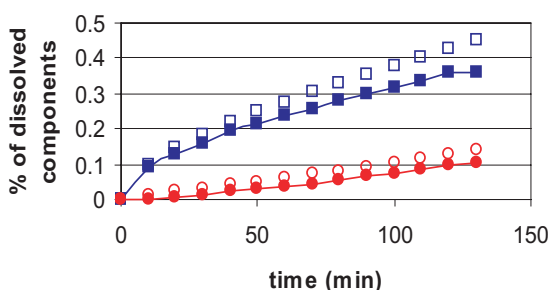

c. Ibuprofen/Maleic acid. 40:60

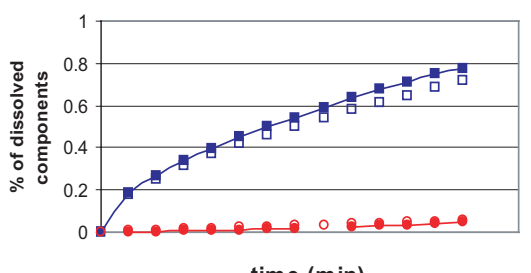

b. Ibuprofen/Succinic acid. (30:70)

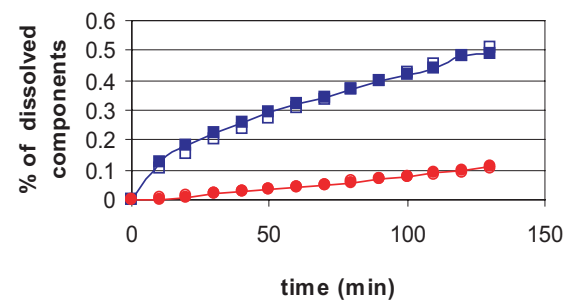

d. Ibuprofen/Maleic acid. (30:70)

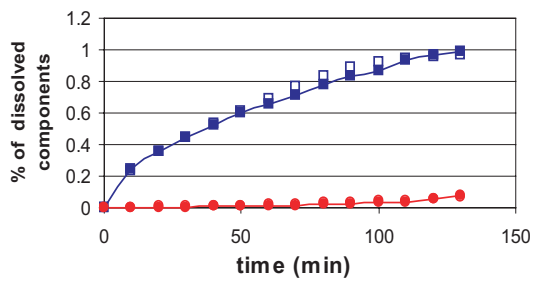

Figure 13: Simulated versus experimental. Continuous lines with filled dots: Dissolution profiles of ibuprofen and excipient from ibuprofen/acid excipient compressed discs. Empty dots: Quantitative simulated results corresponding to the experimental situations. Red: ibuprofen. Blue: acid excipient. The titles show what are the two components in each experiment and the weight ratio in which they are used.

\section{Ibuprofen/Male ic acid (50:50)}

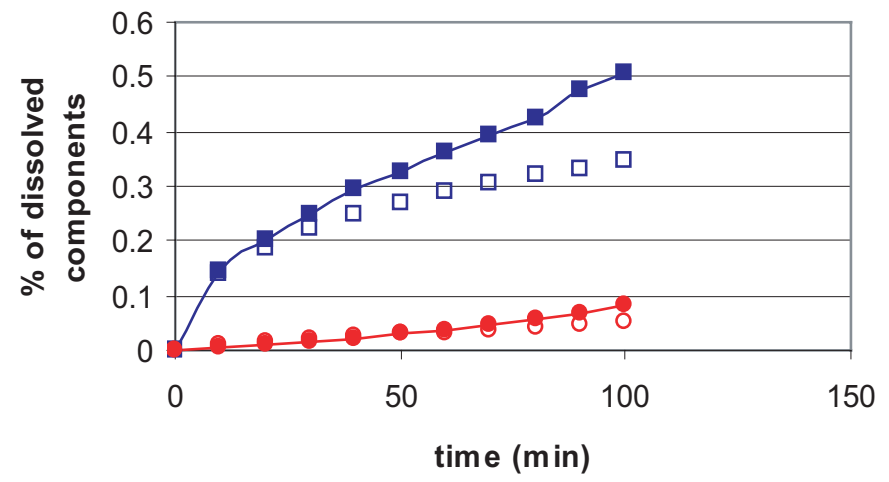

Figure 14: Simulated versus experimental. Continuous lines with filled dots: Dissolution profiles of ibuprofen and excipient from ibuprofen/acid excipient compressed discs. Empty dots: Quantitative simulated results corresponding to the experimental situations. Red: ibuprofen. Blue: maleic acid. 


\section{Acknowledgements:}

The authors would like to acknowledge funding support from Irish National Institute for Cellular Biotechnology (NICB) under the HEA CRTLI initiative, Ireland, as well as Dr. A. M. Healy (School of Pharmacy, Trinity College Dublin), for access to the experimental data, used as reference in this study.

\section{References}

[1] United States Pharmacopoeia. Online homepage available from: http://www.usp.org/frameset.htm. Access date: 16/06/2004.

[2] X. Chen, W. Chen, A. H. Hical, B.-C. Shen, and L. T. Fan. Stochastic modeling of controlleddrug release. Biochemical Engineering Journal, 2:161-177, 1998.

[3] B. Chopard and M. Droz. Cellular Automata Modeling of Physical Systems. Cambridge University Press, 1998.

[4] P. Costa and J. M. S. Lobo. Modeling and comparison of dissolution profiles. European Journal of Pharmaceutical Sciences, 13:123-133, 2001.

[5] J. Cranck. The Mathematics of Diffusion. Clarendon Press, Oxford, 1975.

[6] M. Crane, L. Crane, A. M. Healy, O. I. Corrigan, K. M. Gallagher, and L. McCarthy. A Pohlhausen solution for the Mass Flux From a Multi-layered Compact in the USP Drug Dissolution Apparatus. Simulation Modelling Practice and Theory (SIMPAT), 2004.

[7] M. Crane, N. J. Hurley, L. Crane, A. M. Healy, O. I. Corrigan, K. M. Gallagher, and L. G. McCarthy. Simulation of the USP drug delivery problem using CFD: experimental, numerical and mathematical aspects. Simulation Modelling Practice and Theory (SIMPAT), 12, 2004.

[8] A. Göpferich. Erosion of composite polymer matrices. Biomaterials, 18:397-403, 1997.

[9] A. Göpferich and R. Langer. Modeling monomer release from bioerodible polymers. Journal of Controlled Release, 33:55-69, 1995.

[10] A. M. Healy. Investigations of the dissolution mechanisms of acidic drug-excipient compacts. PhD thesis, University of Dublin, Trinity College, 1995.

[11] A. M. Healy and O. I. Corrigan. Predicting the dissolution rate of ibuprofen-acidic excipient compressed mixtures in reactive media. International Journal of Pharmaceutics, 84:167-173, 1992.

[12] A. M. Healy and O. I. Corrigan. The influence of excipient particle size, solubility and acid strength on the dissolution of an acidic drug from two-component compacts. International Journal of Pharmaceutics, 143:211-221, 1996.

[13] A. Kalampokis, P. Argyrakis, and P. Macheras. Heterogeneous tube model for the study of small intestinal transit flow. Pharmaceutical Research, 16(1), 1999.

[14] K. Kosmidis and P. Argyrakis. Fractal kinetics in drug release from finite fractal matrices. Journal of Chemical Physics, 119(12), 2000.

[15] K. Kosmidis, E. Rinaki, P. Argyrakis, and P. Macheras. Analysis of case ii drug transport with radial and axial release from cylinders. International Journal of Pharmaceutics, 254, 2003.

[16] D. P. Landau and K. Binder. Monte Carlo Simulations in Statistical Physics. Cambridge University Press, 2000. 
[17] L. McCarthy, C. Kosiol, A. Healy, G. Bradley, J. Sexton, and O. Corrigan. Simulating the hydrodynamic conditions in the United States Pharmacopeia paddle dissolution apparatus. AAPS PharmSciTech, 4(2):109-115, 2003.

[18] B. Narasimhan. Mathematical models describing polymer dissolution: consequences for drug delivery. Advanced Drug Delivery Reviews, 48:195-210, 2001.

[19] L. Prandtl and O. Tietjens. Applied Hydro- and Aeromechanics. Dover Publications New York Paperback, 1957. Chapter IV.

[20] Z. Ramtoola and O. I. Corrigan. Dissolution characteristics of benzoic acid and salicylic acid mixtures in reactive media. Drug Development and Industrial Pharmacy, 13:9-11, 1987.

[21] H. Schlichting. Boundary-Layer Theory. McGraw-Hill, New York; London [etc.], 7 edition, 1979. Chap. XII.

[22] J. Siepmann and A. Göpferich. Mathematical modeling of bioerodible, polymeric drug delivery systems. Advanced Drug Delivery Reviews, 48:229-247, 2001.

[23] J. Siepmann and N. A. Peppas. Modeling of drug release from delivery systems based on hydroxypropyl methylcellulose (HPMC). Advanced Drug Delivery Reviews, 48:139-157, 2001.

[24] Y. Sun, Y. Peng, Y. Chen, and A. J. Shukla. Application of artificial neural networks in the design of controlled release drug delivery systems. Advanced Drug Delivery Reviews, 55:1201-1215, 2003. Preface.

[25] K. Zygourakis and P. A. Markenscoff. Computer-aided design of bioerodible devices with optimal release characteristics: a cellular automata approach. Biomaterials, 17:125-135, 1996. 